\title{
ON THE DISTANCE OF THE COMPOSITION OF TWO DERIVATIONS TO THE GENERALIZED DERIVATIONS
}

\author{
by MATEJ BREŠAR
}

(Received 19 June, 1989; revised 11 September, 1989)

Introduction. A well-known theorem of E. Posner [10] states that if the composition $d_{1} d_{2}$ of derivations $d_{1}, d_{2}$ of a prime ring $A$ of characteristic not 2 is a derivation, then either $d_{1}=0$ or $d_{2}=0$. A number of authors have generalized this theorem in several ways (see e.g. [1], [2], and [5], where further references can be found). Under stronger assumptions when $A$ is the algebra of all bounded linear operators on a Banach space (resp. Hilbert space), Posner's theorem was reproved in [3] (resp. [12]). Recently, M. Mathieu [8] extended Posner's theorem to arbitrary $\mathrm{C}^{*}$-algebras.

Let $d_{1}, d_{2}$ be derivations of a normed algebra $A$. Our purpose is to estimate the distance of $d_{1} d_{2}$ to the set of all generalized derivations of $A$. We consider the case when $A$ is an ultraprime normed algebra (Theorem 1), the case when $d_{1}=d_{2}$ and $A$ is ultrasemiprime (Theorem 2), and finally, the case when $A$ is a von Neumann algebra (Theorem 3). As a consequence of these results we obtain a partial answer to Mathieu's question [8]: what is the norm of the composition of two derivations in a prime $\mathrm{C}^{*}$-algebra?

Our results will follow easily from two entirely elementary observations; it is our aim in this paper to point out the kind of method that could be used.

Notation and preliminaries. Let $A$ be a ring and let $a, b \in A$. By $M_{a, b}$ we denote the mapping $x \rightarrow a x b$ on $A$. Recall that $A$ is said to be prime if $M_{a, b}=0$ implies $a=0$ or $b=0$. M. Mathieu [6], [9] introduced the notion of an ultraprime normed algebra: a complex normed algebra is ultraprime if there exists a constant $c>0$ such that

$$
\left\|M_{a, b}\right\| \geq c\|a\|\|b\| \quad \text { for all } a, b \in A \text {. }
$$

Every prime $C^{*}$-algebra $A$ is ultraprime since $\left\|M_{a, b}\right\|=\|a\|\|b\|$ for all $a, b \in A[7$, Proposition 2.3]. Note that $B(X)$, the algebra of all bounded linear operators on a normed space $X$, also has this property; (moreover, the same is true for every subalgebra of $B(X)$ which contains all finite rank operators).

A ring $A$ is said to be semiprime if $M_{a, a}=0$ implies $a=0$. M. Mathieu called a complex normed algebra $A$ ultrasemiprime if there exists a constant $c>0$ such that

$$
\left\|M_{a, a}\right\| \geq c\|a\|^{2} \quad \text { for all } a \in A \text {. }
$$

Every $C^{*}$-algebra $A$ is ultrasemiprime. Namely, for $a \in A$ we have $\left\|a a^{*} a a^{*}\right\|=$ $\left\|\left(a a^{*}\right)^{2}\right\|=\left\|a a^{*}\right\|^{2}=\|a\|^{4}$; hence $\|a\|^{4} \leq\left\|a a^{*} a\right\|\|a\|$, which yields $\left\|a a^{*} a\right\|=\|a\|^{3}$; consequently $\left\|M_{a, a}\right\|=\|a\|^{2}$.

Let $A$ be a ring. An additive mapping $\delta: A \rightarrow A$ is called a generalized inner derivation if $\delta(x)=a x+x b$ for some $a, b \in A$. Note that $\delta(x y)=\delta(x) y+x[y, b]$, where $[u, v]$ denotes the commutator $u v-v u$. Thus

$$
\delta(x y)=\delta(x) y+x h(y) \quad \text { for all } x, y \in A,
$$

where $h$ is an inner derivation of $A$. Now, an additive mapping $\delta: A \rightarrow A$ will be called a generalized derivation if there exists a derivation $h$ of $A$ such that $\delta$ satisfies (3). By $\Delta(A)$

Glasgow Math. J. 33 (1991) 89-93. 
we denote the set of all generalized derivations of $A$. In case $A$ is a normed algebra, $\Delta_{b}(A)$ denotes the set of all $\delta$ in $\Delta(A)$ which are also bounded linear operators on $A$. Next, the set of all derivations of a ring $A$ will be denoted by $D(A)$, and by $D_{b}(A)$ we denote the set of all bounded derivations of a normed algebra $A$.

Remark 1. Let $A$ be a ring, $h: A \rightarrow A$ be any function, and $\delta: A \rightarrow A$ be an additive mapping satisfying (3). We intend to show that under rather mild assumptions $h$ must necessarily be a derivation. On the one hand we have

$$
\delta(x y z)=\delta(x(y z))=\delta(x) y z+x h(y z),
$$

and on the other hand,

$$
\delta(x y z)=\delta((x y) z)=\delta(x) y z+x h(y) z+x y h(z) .
$$

Comparing these two expressions we obtain

$$
x(h(y z)-h(y) z-y h(z))=0(x, y, z \in A) .
$$

Similarly, by computing $\delta(x(y+z))$ in two ways, one shows that

$$
x(h(y+z)-h(y)-h(z))=0(x, y, z \in A) .
$$

Thus, if $A$ has the property that $A a=0$ implies $a=0$, in particular, if $A$ is semiprime, then $h$ is a derivation.

Remark 2. Suppose a ring $A$ has a unit element 1 and take $x=1$ in (3). Then we get $\delta(y)=\delta(1) y+h(y)$ for all $y \in A$. Hence we see that every generalized derivation of $A$ is an inner generalized derivation if and only if every derivation of $A$ is inner.

We now state the crucial observations, which can be proved by direct computations.

Observation 1. Let $A$ be a ring, $d_{1}, d_{2} \in D(A)$ and $\delta \in \Delta(A)$. A mapping $F=$ $d_{1} d_{2}-\delta$ then satisfies the identity

$$
F(x y z)-F(x y) z-x F(y z)+x F(y) z=d_{1}(x) y d_{2}(z)+d_{2}(x) y d_{1}(z) .
$$

ObServation 2. Let $A$ be $a$ ring, and let $f: A \rightarrow A, g: A \rightarrow A$ be arbitrary functions. Then for all $x, y, z, w, u \in A$, we have

$$
\begin{aligned}
2 f(x) y g(z) w f(u)= & \{f(x) y g(z)+g(x) y f(z)\} w f(u)+f(x) y\{g(z) w f(u) \\
& +f(z) w g(u)\}-\{f(x)(y f(z) w) g(u)+g(x)(y f(z) w) f(u)\} .
\end{aligned}
$$

Remark 3. Using the above observations it is easy to prove the following slight generalization of Posner's theorem: if $d_{1}, d_{2} \in D(A)$ and $d_{1} d_{2} \in \Delta(A)$, where $A$ is a prime ring of characteristic not 2 , then either $d_{1}=0$ or $d_{2}=0$. In our forthcoming paper [2] some similar results can be found.

The results. Let $A$ be a normed algebra and let $d_{1}, d_{2}, d \in D_{b}(A)$. Our purpose is to estimate $\operatorname{dist}\left(d_{1} d_{2}, \Delta_{b}(A)\right)$ and $\operatorname{dist}\left(d^{2}, \Delta_{b}(A)\right)$, where

$$
\operatorname{dist}\left(d_{1} d_{2}, \Delta_{b}(A)\right)=\inf \left\{\left\|d_{1} d_{2}-\delta\right\|, \delta \in \Delta_{b}(A)\right\} .
$$

THEOREM 1. Let $A$ be an ultraprime normed algebra, and let $d_{1}, d_{2} \in D_{b}(A)$. If a constant $c>0$ satisfies (1), then

$$
\operatorname{dist}\left(d_{1} d_{2}, \Delta_{b}(A)\right) \geq\left(c^{2} / 6\right)\left\|d_{1}\right\|\left\|d_{2}\right\| .
$$


Proof. Take $\delta \in \Delta_{b}(A)$. According to Observation 1 we have

$$
\left\|d_{1}(x) y d_{2}(z)+d_{2}(x) y d_{1}(z)\right\| \leq 4\left\|d_{1} d_{2}-\delta\right\|\|x\|\|y\|\|z\|
$$

for all $x, y, z \in A$. Using this relation and Observation 2 we then get

$$
\begin{aligned}
2\left\|d_{1}(x) y d_{2}(z) w d_{1}(u)\right\| \leq & \left(4\left\|d_{1} d_{2}-\delta\right\|\|x\|\|y\|\|z\|\right)\|w\|\left\|d_{1}(u)\right\| \\
& +\left\|d_{1}(x)\right\|\|y\|\left(4\left\|d_{1} d_{2}-\delta\right\|\|z\|\|w\|\|u\|\right) \\
& +4\left\|d_{1} d_{2}-\delta\right\|\|x\|\left\|y d_{1}(z) w\right\|\|u\| \\
\leq & 12\left\|d_{1} d_{2}-\delta\right\|\left\|d_{1}\right\|\|x\|\|y\|\|z\|\|w\|\|u\| .
\end{aligned}
$$

That is,

$$
\left\|M_{d_{1}(x), d_{2}(z) w d_{1}(u)}\right\| \leq 6\left\|d_{1} d_{2}-\delta\right\|\|x\|\|z\|\|w\|\|u\| .
$$

Thus it follows that

$$
c\left\|d_{1}(x)\right\|\left\|d_{2}(z) w d_{1}(u)\right\| \leq 6\left\|d_{1} d_{2}-\delta\right\|\left\|d_{1}\right\|\|x\|\|z\|\|w\|\|u\| .
$$

Hence $c\left\|d_{2}(z) w d_{1}(u)\right\| \leq 6\left\|d_{1} d_{2}-\delta\right\|\|z\|\|w\|\|u\|$ from which

$$
c^{2}\left\|d_{2}(z)\right\|\left\|d_{1}(u)\right\| \leq 6\left\|d_{1} d_{2}-\delta\right\|\|z\|\|u\|
$$

is derived. Consequently $c^{2}\left\|d_{1}\right\|\left\|d_{2}\right\| \leq 6\left\|d_{1} d_{2}-\delta\right\|$. The proof of the theorem is complete.

TheOREM 2. Let $A$ be an ultrasemiprime normed algebra, and let $d \in D_{b}(A)$. If a constant $c>0$ satisfies (2), then

$$
\operatorname{dist}\left(d^{2}, \Delta_{b}(A)\right) \geq(c / 2)\|d\|^{2} .
$$

Proof. Take $\delta \in \Delta_{b}(A)$. By Observation 1 we have

$$
2\|d(x) y d(x)\| \leq 4\left\|d^{2}-\delta\right\|\|x\|^{2}\|y\|(x, y \in A) .
$$

It is easy to see that this relation implies the assertion of the theorem.

Let $A$ be a von Neumann algebra. Since every derivation of $A$ is inner [11, Theorem 4.1.6] it follows from Remark 2 that every generalized derivation of $A$ is an inner generalized derivation. In particular, $D_{b}(A)=D(A)$ and $\Delta_{b}(A)=\Delta(A)$.

Theorem 3. Let $A$ be a von Neumann algebra. If $d_{1}, d_{2} \in D(A)$, then

$$
\operatorname{dist}\left(d_{1} d_{2}, \Delta(A)\right) \leq(1 / 2)\left\|d_{1}\right\|\left\|d_{2}\right\| \text {. }
$$

In particular, for every $d \in D(A)$,

$$
\operatorname{dist}\left(d^{2}, \Delta_{b}(A)\right)=(1 / 2)\|d\|^{2} .
$$

Proof. Since $A$ is a von Neumann algebra there exist $a_{1}, a_{2} \in A$ such that $d_{i}(x)=$ $\left[a_{i}, x\right](x \in A)$. For arbitrary $c_{1}, c_{2}$ in $Z$, the center of $A$, define $\delta \in \Delta(A)$ by

$$
\delta(x)=\left(a_{1} a_{2}+c_{1} c_{2}-c_{2} a_{1}-c_{1} a_{2}\right) x+x\left(a_{2} a_{1}+c_{1} c_{2}-c_{2} a_{1}-c_{1} a_{2}\right)
$$

Then, $d_{1} d_{2}-\delta=-M_{a_{1}-c_{1}, a_{2}-c_{2}}-M_{a_{2}-c_{2}, a_{1}-c_{1}}$ and therefore

$$
\left\|d_{1} d_{2}-\delta\right\|=\left\|M_{a_{1}-c_{1}, a_{1}-c_{2}}+M_{a_{2}-c_{2}, a_{1}-c_{1}}\right\| \leq 2\left\|a_{1}-c_{1}\right\|\left\|a_{2}-c_{2}\right\| .
$$


Since $\left\|d_{i}\right\|=2 \operatorname{dist}\left(a_{i}, Z\right)[4],[13]$ we conclude that

$$
\operatorname{dist}\left(d_{1} d_{2}, \Delta(A)\right) \leq 2 \operatorname{dist}\left(a_{1}, Z\right) \operatorname{dist}\left(a_{2}, Z\right)=(1 / 2)\left\|d_{1}\right\|\left\|d_{2}\right\| .
$$

This inequality, together with Theorem 2 and the fact that $\left\|M_{a, a}\right\|=\|a\|^{2}$ for all $a \in A$, yields the second assertion.

Remark 4. As we have mentioned above, in [8] M. Mathieu posed the following question: what is the norm of the composition of two derivations of a prime $\mathrm{C}^{*}$-algebra? The results above enable us to discuss this problem. Since every $\mathrm{C}^{*}$-algebra $A$ satisfies $\left\|M_{a, a}\right\|=\|a\|^{2}$ for all $a \in A$, it follows from Theorem 2 that for every $d \in D_{b}(A)$, we have

$$
(1 / 2)\|d\|^{2} \leq\left\|d^{2}\right\| \leq\|d\|^{2} \text {. }
$$

This estimate cannot be improved. Indeed, let $A$ be the algebra of $2 \times 2$ matrices over the complex field $\mathbb{C}$, and let

$$
p=\left[\begin{array}{ll}
1 & 0 \\
0 & 0
\end{array}\right], \quad n=\left[\begin{array}{ll}
0 & 1 \\
0 & 0
\end{array}\right] .
$$

Define derivations $d$ and $g$ by $d(x)=[p, x], g(x)=[n, x]$. Note that $d=d^{3}$; hence $\|d\| \leq\|d\|\left\|d^{2}\right\|$ which means that $\left\|d^{2}\right\| \geq 1$. Using the fact that $\|d\|=2 \operatorname{dist}(p, \mathbb{C} 1)$, or otherwise, one shows that $\|d\|=1$. But then $\left\|d^{2}\right\|=\|d\|^{2}$. Next, we claim that $\left\|g^{2}\right\|=(1 / 2)\|g\|^{2}$. Namely, observe that $\|g\|=2$; since $g^{2}=-2 M_{n, n}$ we have $\left\|g^{2}\right\|=2$.

Now let $A$ be a prime $C^{*}$-algebra. As we have mentioned above, in this case $\left\|M_{a, b}\right\|=\|a\|\|b\|$ for all $a, b \in A$. Thus it follows from Theorem 1 that for all $d_{1}, d_{2} \in D_{b}(A)$,

$$
(1 / 6)\left\|d_{1}\right\|\left\|d_{2}\right\| \leq\left\|d_{1} d_{2}\right\| \leq\left\|d_{1}\right\|\left\|d_{2}\right\| .
$$

We leave as an open question whether or not the constant $1 / 6$ can be improved.

ACKNowledgements. I wish to express my thanks to Dr. B. Magajna for helpful conversations. Also I thank the referee for his valuable suggestions.

\section{REFERENCES}

1. J. Bergen, I. N. Herstein and J. Kerr, Lie ideals and derivations of prime rings, J. Algebra 71 (1981), 259-267.

2. M. Brešar and J. Vukman, Orthogonal derivations and an extension of a theorem of Posner, to appear in Rad. Mat.

3. C. K. Fong and A. R. Sourour, On the operator identity $\sum A_{k} X B_{k} \equiv 0$, Canad. J. Math. 31 (1979), 845-857.

4. P. Gajendragadkar, Norm of a derivation on a von Neumann algebra, Trans. Amer. Math. Soc. 170 (1972), 165-170.

5. C. Lanski, Differential identities, Lie ideals, and Posner's theorems, Pacific J. Math. 134 (1988), 275-297.

6. M. Mathieu, Applications of ultraprime Banach algebras in the theory of elementary operators, Thesis (Tübingen, 1986). 223-244.

7. M. Mathieu, Elementary operators on prime C*-algebras I, Math. Ann. 284 (1989),

8. M. Mathieu, Properties of the product of two derivations of a $\mathrm{C}^{*}$-algebra, to appear in Canad. Math. Bull. 
9. M. Mathieu, Rings of quotients of ultraprime Banach algebras. With applications to elementary operators, to appear in the Proceedings of the Conference on Banach algebras and Automatic Continuity (Canberra, Australia, 1989).

10. E. Posner, Derivations in prime rings, Proc. Amer. Math. Soc. 8 (1957), 1093-1100.

11. S. Sakai, $C^{*}$-algebras and $W^{*}$-algebras (Springer-Verlag, 1971).

12. J. P. Williams, On the range of a derivation, Pacific J. Math. 38 (1971), 273-279.

13. L. Zsido, The norm of a derivation in a W*-algebra, Proc. Amer. Math. Soc. 38 (1973), $147-150$.

Institute of Mathematics, Physics and Mechanics

UNIVERSITY OF LJUBLJANA

P.O. Box 543

61111 LJUBLJANA

Yugoslavia 